\title{
Morphological studies of five known nematode species via taxa-informatics approaches
}

\author{
Alka Dubey ", Vinita Sharma \\ Zoological Survey of India, Northern Regional Centre Dehradun, Uttarakhand, INDIA \\ Email address: \\ Alkabioinfo964@gmail.com (A. Dubey)
}

To cite this article:

Alka Dubey, Vinita Sharma. Morphological Studies of Five Known Nematode Species Via Taxa-Informatics Approaches. Science Innovation. Vol. 2, No. 1, 2014, pp. 7-10. doi: 10.11648/j.si.20140201.12

\begin{abstract}
Last few decades our generation running in an advance biological research in each and every field related to life sciences. Now time to change taxonomy to taxa informatics. The term of taxa informatics is that to solve the taxonomical complexity with the help of computer hardware's and software's with well developed computational programmes so that's phenomenon known as taxa-informatics. In this term taxa relate with taxonomy and informatics related to computational biology. Finally in this research identified five known species Mylonchulus (Mylonchulidae : Mononchida) with diversify morphological changes in taxonomic calculations.
\end{abstract}

Keywords: Taxainformatics, Diversify, Nematodes, Mylonchulus, Morphology

\section{Introduction}

Nematodes are diverse metazoans with an estimated total number of a million species. They are arguably the most numerous metazoans in soil and aquatic sediments. From an environmental point of view, nematodes are part of nearly all ecosystems and their roles as bacterivores, herbivores, parasites of animals and plants, and consumers of dissolved as well as particulate organic matter. They have critical roles in the flow of energy and cycling of nutrients. From an anthropocentric point of view, they parasitize humans and plants, domestic and wild animals and they can serve as indicators of environmental change (Lambshead, 2004). An attempt has been made on 5 species of Mylonchulus reported from RNP Uttarakhand for the above study.

The most nematode species are identified using a number of morphometric and morphological characters and the morphometric characters are particularly suitable for measurement and analysis by a computer. Also, they point out some computer identification programs based on the nematode's morphometric characters, have already been written for identifying genus Helicotylenchus and Longidorus. (Peet et al., 1990) developed a digital imaging system for measuring various physical characteristics of individual nematodes and for comparing groups of nematodes. (La Blanca et al., 1992) developed computational procedures for estimating and calibrating nematode features from digital images. As they point out, feature identification from digitized microscopic images has been used for more than 10 years but is relatively new in nematology. (Bravo and Roca, 1994) wrote a program for identifying genus Xiphinema using dBASE IV software and (Robbins and Brown 1994) did the same to genus Longidorus using LOTUS.

\section{Material and Methods}

For this analysis so many type of approaches are needed as technique and as microscopic instruments also. Before this process collected soil samples from arouns the root of forest tree from protected areas, then Plant and soil nematodes were extracted from soil samples by a combination of sieving and decantation technique. The extracted nematodes were killed and fixed in hot $4 \%$ formalin then nematode kept in glass tubes and nematodes were transferred from fixative to a solution of 5 parts of glycerine and 95 parts of $30 \%$ alcohol in a cavity block and the cavity block having the nematodes will be placed in desiccators with Calcium Chloride for dehydration of nematodes at room temperature for 2-3 weeks. Then nematodes were finally mounted on a glass slide in pure anhydrous glycerine and sealed with wax. Measurements and drawings were made by using Olympus BX51 with digital camera, compound microscope, and camera Lucida, For the study of taxainformatic using computational biology via 64 bit operating system window 7 , software's and hard wares. 


\section{Results and Discussion}

Taxonomic analysis: The nematode yielded from analysis were identified as 5 known species of genus Mylonchulus as given in tables 1 to 5 . All these species well known but according present observation so many type of morphological diversity are found with taxonomic calculations but in a view of taxainformatics these identified species are design in computational plate form as phylogeny and identifying morphological characteristic programming for this order. As a type of software. According their species evolution in taxonomic field as given in figure.

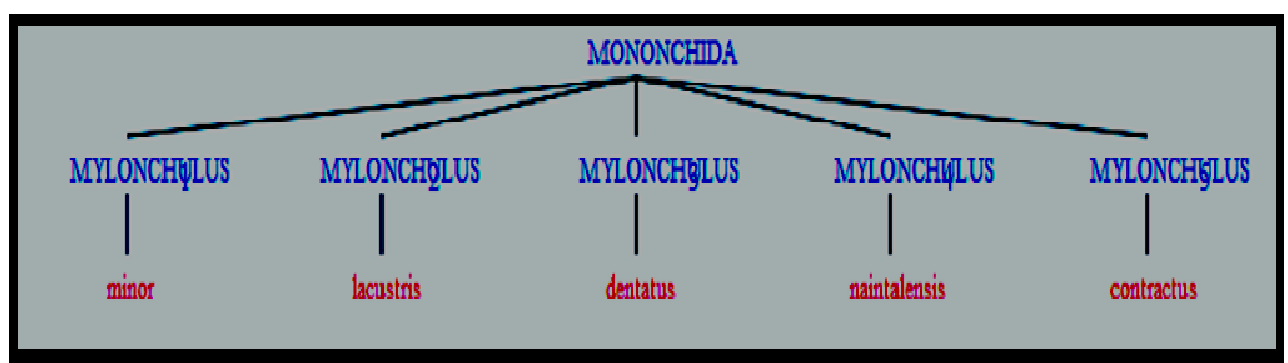

Fig 1. all species are arranged basis on their taxonomic evolutions

In this figure first node explains the order and second node explains genus Mylonchulus and third node explains

the name of species.

Table No 1. Taxonomic morphological description for MYLONCHULUS NAINITALENSIS JAIRAJPURI, 1970

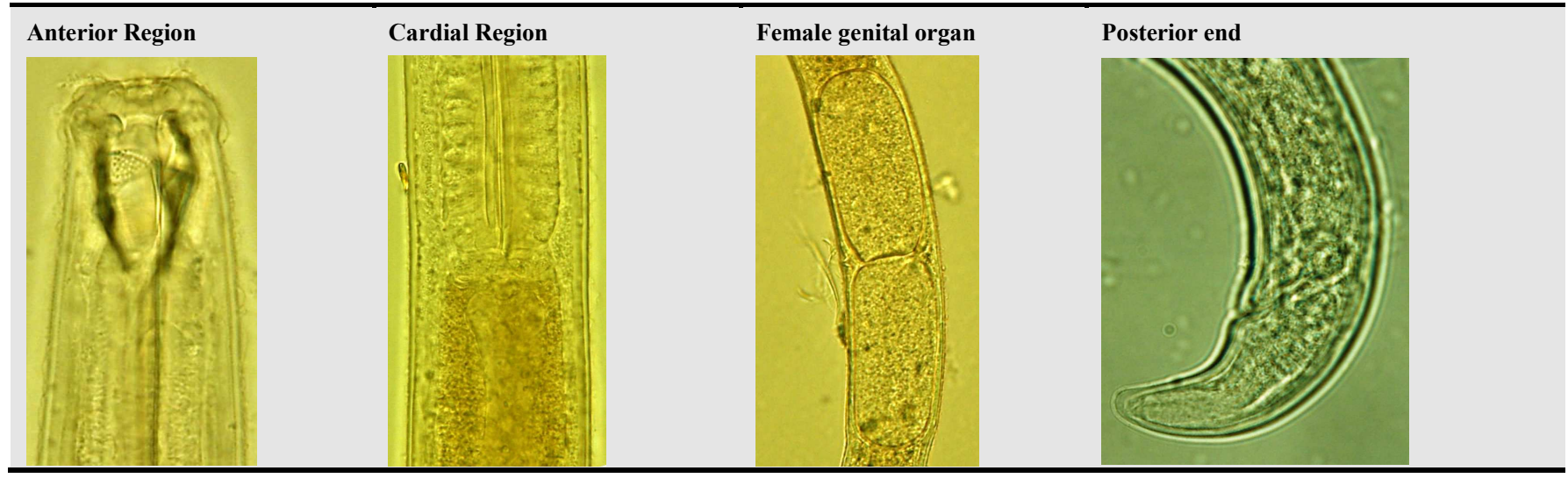

Morphological taxonomic calculation of female: $\mathrm{L}=1.1 \mathrm{~mm} ; \mathrm{a}=25 ; \mathrm{b}=3.0 ; \mathrm{c}=24 ; \mathrm{c}^{\prime}=1.6, \mathrm{~V}=65$

Description: Lip region $25 \mu \mathrm{m} \times 10 \mu \mathrm{m}$. Buccal cavity $23 \mu \mathrm{m} \times 14 \mu \mathrm{m}$. Dorsal tooth massive, its apex at $19 \mu \mathrm{m}$ or $83 \%$ of the length of buccal cavity from base. Subventral walls with 6 trasverse rows of denticles. Submedian teeth present. Reproductive system amphidelphic. Caudal glands tandem. Tail bluntly conoid. Spinneret subterminal dorsally.

Table No 2. Taxonomic morphological description for MYLONCHULUS LACUSTRIS (N.A. COBB IN M.V. COBB,1915) ANDRASSY, 1958

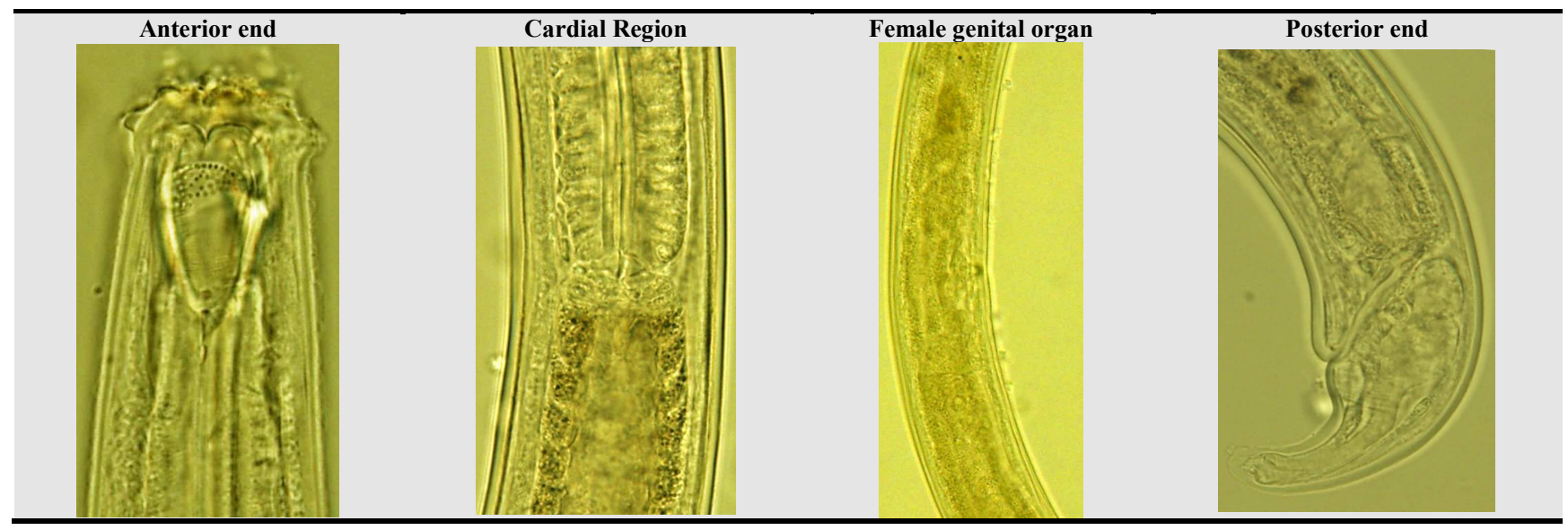

Morphological taxonomic calculation of female: $\mathrm{L}=1.1 \mathrm{~mm} ; \mathrm{a}=27 ; \mathrm{b}=3.3 ; \mathrm{c}=29 ; \mathrm{c}^{\prime}=1.5 ; \mathrm{V}=59$

Description: Lip region $24 \mu \mathrm{m} \times 9 \mu \mathrm{m}$. Buccal cavity $23 \mu \mathrm{m} \times 14 \mu \mathrm{m}$. Dorsal tooth massive, its apex at $18 \mu \mathrm{m}$ or $78 \%$ of the length of buccal cavity from base. Sub ventral wall with 7 transverse rows of denticles. Sub median teeth well developed. Reproductive system amphidelphic. Tail conoid accurate. Caudal glands tandem. Spinneret terminal. 
Table No 3. Taxonomic morphological description for MYLONCHULUS DENTATUS JAIRAJPURI. 1970

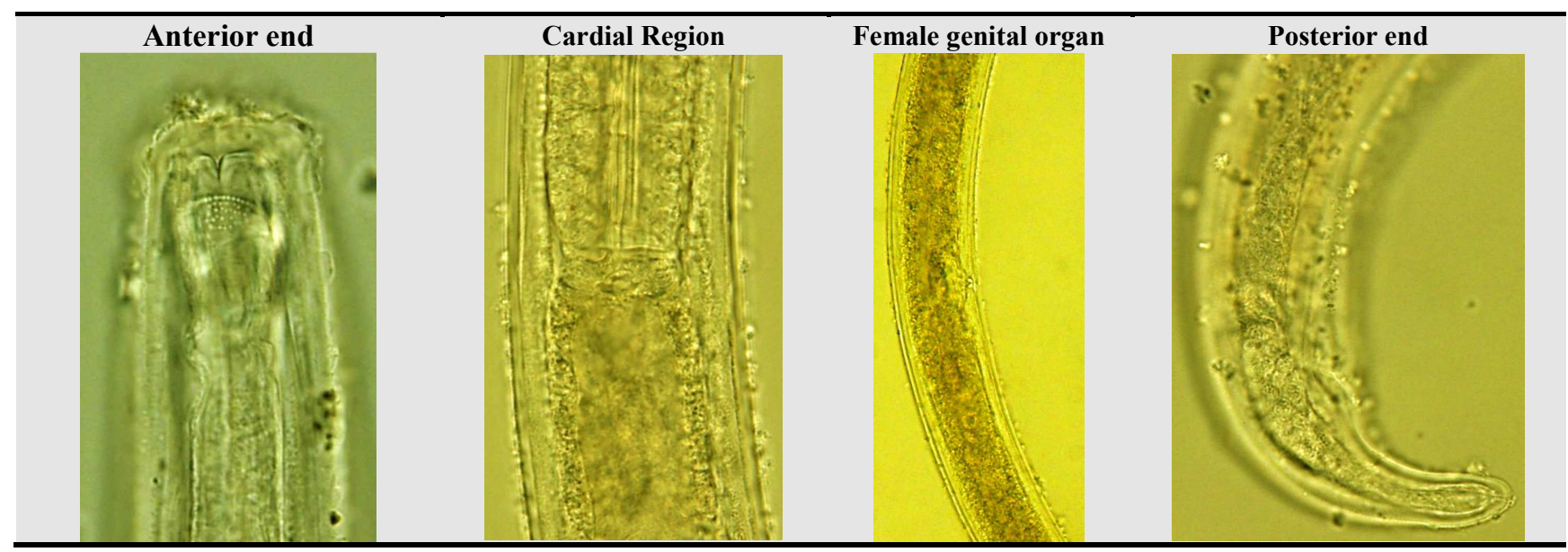

Morphological taxonomic calculation of female: $\mathrm{L}=1.0 \mathrm{~mm} ; \mathrm{a}=29 ; \mathrm{b}=3.5 ; \mathrm{c}=31 ; \mathrm{c}^{\prime}=1.5 ; \mathrm{V}=64$

Description: Lip region $23 \mu \mathrm{m}$ x $9 \mu \mathrm{m}$. Buccal cavity $23 \mu \mathrm{m}$ x $14 \mu \mathrm{m}$. Dorsal tooth massive, its apex at $18 \mu \mathrm{m}$ or $78 \%$ of the length of buccal cavity from base. Subventral wall with transverse rows of denticles, not arranged in definite rows. Submedian teeth prominent. Reproductive system amphidelphic. Tail elongated conoid, Caudal glands grouped. Spinneret terminal.

Table No 4. Taxonomic morphological description for MYLONCHULUS CONTRACTUS JAIRAJPURI,1970

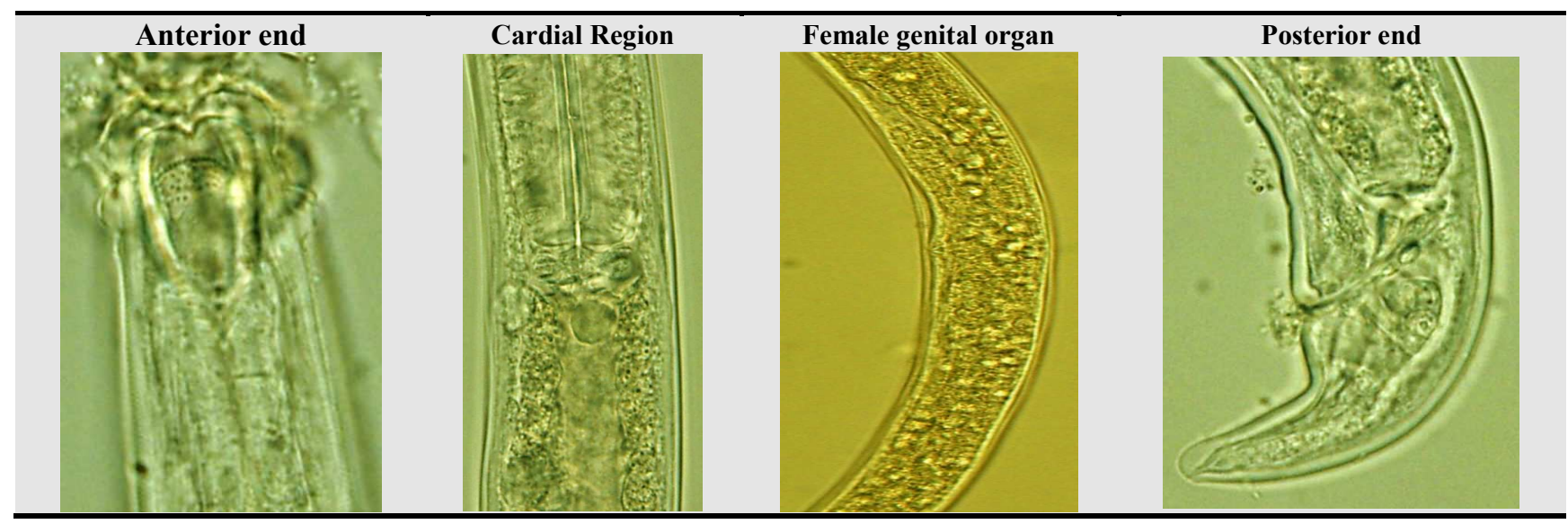

Morphological taxonomic calculation of female $: \mathrm{L}=0.91 \mathrm{~mm} ; \mathrm{a}=31 ; \mathrm{b}=3.2 ; \mathrm{c}=28 ; \mathrm{c}^{\prime}=1.5, \mathrm{~V}=61$

Description: Lip region $19 \mu \mathrm{m}$ x $89 \mu \mathrm{m}$. Buccal cavity $17 \mu \mathrm{m}$ x $10 \mu \mathrm{m}$. Dorsal tooth medium, its apex at $14 \mu \mathrm{m}$ or $82 \%$ of the length of buccal cavity from base. Subventral walls with 5 transverse rows of denticles. Submedian teeth present. Reproductive system amphidelphic. Tail bluntly conoid, Caudal glands grouped. Spinneret subterminal dorsally.

Table No 5. Taxonomic morphological description for MYLONCHULUS MINOR (COBB,1893) ANDRASSY 1958

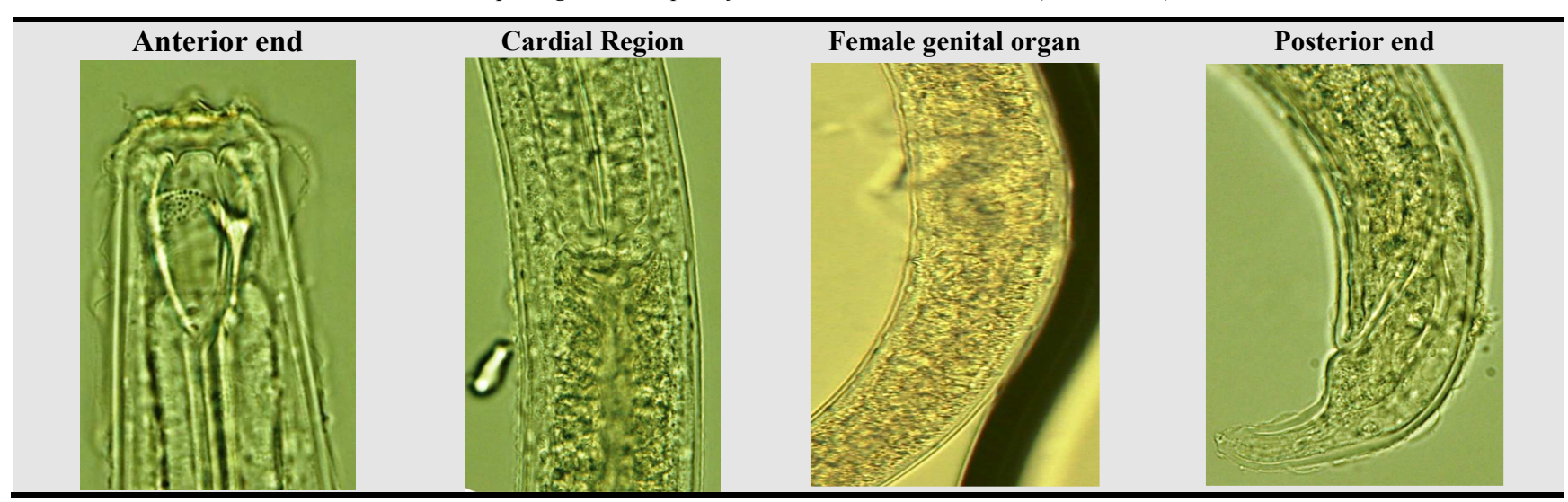

Morphological taxonomic calculation Female: $\mathrm{L}=1.1 \mathrm{~mm} ; \mathrm{a}=32 ; \mathrm{b}=3.2 ; \mathrm{c}=28 ; \mathrm{c}^{\prime}=1.6, \mathrm{~V}=60$

Description: Lip region $20 \mu \mathrm{m}$ x $8 \mu \mathrm{m}$. Buccal cavity $24 \mu \mathrm{m}$ x $15 \mu \mathrm{m}$. Dorsal tooth medium, its apex at $18 \mu \mathrm{m}$ or $75 \%$ of the length of buccal cavity from base.

Subventral walls with 5 transverse rows of denticles. Submedian teeth present. Reproductive system amphidelphic. Tail arcuate conoid with round terminus. Caudal gland tandem. Spinneret terminal. 


\section{Conclusion}

Finally study with this entitled: Morphological studies of five known nematodes species via Taxa-informatics approaches. Firstly this is new scientific word in a field of taxonomy from India really this will be helpful for identifying known and unknown type of species with time saving and good percentage of accuracy in this taxonomic scenario. And in this observation all 5 species of Mylonchulus recorded first time from Rajaji National Park, Uttarakhand and all specimens have been registered and deposited with the National Zoological Collection, NRC, ZSI, Dehradun, for this analysis so many type of evidence support this theory basis on their scientific referencing.

\section{Acknowledgments}

The authors wish to thanks Director, Zoological Survey of India, Kolkata and the Officer -in-Charge, NRC, ZSI, Dehradun for proving necessary facilities and authors also grateful for Uttarakhand State Council for Science and Technology, DehraDun for funding this scientific research project.

\section{References}

[1] Lambshead, P.J.D. (2004). Marine nematode biodiversity. In: Chen, Z.X., Chen, SY \& Dickson D.W. (eds.). Nematology, Advances and Perspectives ACSE-TUP Book Series, pp. 436-467.

[2] Peet, F.G., T.S. Panesar, T.S. Sahota \& J.R. Sutherland (1990) A Digital Image Analysis System for Comparing Groups of Small Nematodes. In: Journal of Nematology 22(3) : 407-413.

[3] La Blanca, N.P., J.F. Valdivia, P. Castillo \& A.G. Barcina (1992). Detecting Nematode Features from Digital Images. In: Journal of Nematology 24 (2) : 289-297.

[4] Bravo, M.A. \& F. Roca (1994). Using dBASE for Identification of Species of the Genus Xiphinema.In: 22nd International Symposium Proceedings of the European Society of Nematologists, Ghent,Belgium, Aug/1994.

[5] Robbins, R.T. \& D.J.F. Brown (1994). A Computer Program "LONG-SORT" to Assist with the Diagnosis and Identification of longidorus Species. In: 22nd International Symposium, Proceedings of the European Society of Nematologists, Ghent, Belgium. 